\title{
El cuidado humanizado y la percepción del paciente en el Hospital EsSalud Huacho. Octubre de 2010
}

\author{
Humanized Care and the Perception of Patient \\ in the Hospital EsSalud Huacho. October 2010
}

\begin{abstract}
Leonor Espinoza Medalla*, Karim Huerta Barrenechea**, Junior Pantoja Quiche**, Walter Velásquez Carmona**, Denisse Cubas Cubas**, Alí Ramos Valencia**.
\end{abstract}

http://dx.doi.org/10.21503/CienciayDesarrollo.2011.v13.05

\section{RESUMEN}

El presente estudio muestra la percepción del cuidado humanizado de los pacientes hospitalizados en el Hospital Gustavo Lanatta Luján de EsSalud Huacho, durante octubre de 2010. En la siguiente investigación descriptiva tipo transversal se realizó una encuesta a 65 pacientes de ambos sexos mayores de 18 años, con un tiempo mayor de 24 horas de hospitalización en los servicios de medicina, obstetricia y cirugía. Los resultados muestran que algunas veces se percibe un $44 \%$ de apoyo en el cuidado, un 47,7\% de apoyo emocional, un 35,4\% de apoyo físico, un 32,2\% de cualidades de enfermería, un 30,8\% de proactividad, un $52,3 \%$ de empatía y un $55,4 \%$ de disponibilidad en el cuidado. Un 36,9\% nunca percibió una priorización en su cuidado. Se concluye, pues, que existe una baja percepción del cuidado humanizado por parte del profesional de enfermería hacia los pacientes, lo que evidencia una atención de baja calidad.

Palabras clave: cuidado, percepción, cuidado humanizado, percepción del paciente.

\section{ABSTRACT}

This study shows the perception of humanized care of patients hospitalized in Lanatta Gustavo Luján of EsSalud Huacho during October 2010. In the following cross sectional descriptive study, we made a survey of 65 patients over 18 years, longer than 24 hours of hospitalization in the Department of Medicine, Obstetrics and Surgery, of both sexes. The results show that sometimes is perceived, $44 \%$ support in the care, emotional support $47,7 \%, 35,4 \%$ physical support, a $32,2 \%$ qualities of nursing proactivity $30,8 \%, 52,3 \%$ and $55,4 \%$ empathy availability in care. $36,9 \%$ never perceived to be prioritized in your care. We conclude that there is a low perception of humanized care at the nurse to patients showing a low quality care.

Keywords: care, perception, humanized care, patient perception.

(C) 2011 All rights reserved.

DOI: http://dx.doi.org/10.21503/CienciayDesarrollo.2011.v13.05

* Docente de la E.A.P. de Enfermería de la UAP, Filial Huacho.

** Alumnos de la E.A.P. de Enfermería de la UAP, Filial Huacho. 


\section{INTRODUCCIÓN}

La enfermería como parte integrante del equipo de salud se ha ido adaptando a los cambios que se han producido en la sanidad, $y$, como no podía ser de otra manera, ha ido asimilando técnicas cada vez más sofisticadas y complejas que en ocasiones la alejan de una visión del hombre como persona que debiera ser la medida de todo, pero que, lamentablemente, se convierte, muchas veces en un extraño en el mundo que precisamente ha sido creado para él en el medio sanitario (1).

El cuidado humanizado se apoya en el conocimiento científico, la capacidad técnica y la relación terapéutica que el enfermero establece con el paciente, y supone acoger al otro de una manera cálida sin dejar de ser uno mismo, despojándose de todos los factores externos que en algún momento puedan afectar la atención comprometida y de óptima calidad que implica el cuidado humanizado (2).

Sin embargo, diversos estudios realizados a nivel nacional e internacional concluyen en que se necesita un mejoramiento en la atención brindada por el personal de enfermería, toda vez que las enfermeras no prestan la debida importancia a la interacción de inicio, proceso y salida tendientes a lograr la satisfacción y recuperación óptima tanto del paciente como de sus familiares.

En su primer libro, Nursing: The Philosophy and Science of Caring, Watson rescata los comportamientos que conforman cada categoría de cuidado así como su significado a la luz de la Teoría del Cuidado Humano (4): Categoría sentimientos delpaciente, Categoría apoyo emocional, Categoria apoyo fisico, Categoría caracteristicas de la enfermera, Categoria cualidades del hacer de la enfermera, Categoría proactividad, Categoría priorizar al ser cuidado, Categoría empatía y Categoria disponibilidad para la atención.
ElHospital Gustavo Lanatta Luján de Huacho es una entidad de nivel II, donde los cuidados de los usuarios hospitalizados es responsabilidad del Departamento de Enfermería, el cual debe trabajar de manera continua por el bienestar de los usuarios y la sociedad a través de la prestación de servicios de excelente calidad desde que el usuario ingresa y durante toda su estancia hospitalaria. Sin embargo, a menudo solemos escuchar denuncias de maltrato, inequidad, negligencia y falta de vocación, entre otras, hechas al equipo de enfermería y al personal médico.

Por ello, creemos que el presente estudio resulta pertinente, pues permitirá indagar acerca de la percepción del cuidado que tienen los pacientes sobre el personal de enfermería. El objetivo es que el mismo sirva a las instituciones formadoras de profesionales y prestadoras de servicios de salud, a fin de mejorar la prestación del servicio y lograr una atención adecuada, buscando el bienestar tanto de los pacientes como de sus familias, es decir, de la comunidad en general.

\section{Problema}

El estudio atiende a la interrogante central: ¿cómo percibe el paciente el cuidado humanizado del profesional de enfermería de EsSalud Huacho durante octubre de 2010?

\section{Objetivo general}

Se centró en describir la percepción del cuidado humanizado en pacientes atendidos por el profesional de enfermería en EsSalud Huacho en octubre de 2010.

\section{Objetivos específicos}

En el caso de los objetivos específicos, se tomaron en consideración los siguientes: 
a) Describir los comportamientos del cuidado.

b) Identificar el cuidado humanizado percibido por los usuarios del servicio de hospitalización.

c) Describir la aplicación de los principios bioéticos en la atención del profesional de enfermería a los pacientes hospitalizados en EsSalud durante el mes de octubre del año 2010.

\section{Hipótesis general}

Se plantea que el paciente hospitalizado en EsSalud Huacho no percibió un cuidado humanizado de los profesionales de enfermería durante octubre del año 2010.

\section{Hipótesis secundarias}

Se plantearon las siguientes:

a) Los profesionales de enfermería que laboran en EsSalud Huacho aplican muy pocas veces un cuidado humanizado.

b) Existe diferencia entre los servicios de hospitalización, de acuerdo al trato percibido por los usuarios.

c) El profesional de enfermería no aplicó los principios bioéticos en la atención al paciente hospitalizado en EsSalud durante el mes de octubre del año 2010.

\section{MATERIAL Y MÉTODO}

Corresponde a un estudio descriptivo, prospectivo y transversal.

Población. Estuvo conformada por un total de 65 pacientes, que correspondan al 100\% de los pacientes hospitalizados durante el mes de octubre del 2010 en los servicios de medicina, cirugía y obstetricia del Hospital Gustavo Lanatta Luján de EsSalud Huacho.

Muestra. Según el análisis, la muestra constituye la población total que aceptó voluntariamente participar en el estudio, esto es, la población y muestra son el $100 \%$ de pacientes hospitalizados en los servicios de medicina, cirugía y gineco-obstetricia del hospital EsSalud Huacho.

Técnicas e instrumentos. Para llevar a efecto la investigación, se utilizó el método de encuesta, y como instrumento, el cuestionario de preguntas cerradas y respuestas politómicas.

\section{RESULTADOS}

Se estudiaron las encuestas realizadas a los pacientes sobre la percepción de las categorías del cuidado humanizado, por género y servicios. Finalmente, se consideró la frecuencia de los pacientes según las categorías evaluadas en los diferentes servicios de hospitalización.

\section{Tabla 1. Pacientes por servicio.}

\begin{tabular}{|c|c|c|c|c|c|}
\hline \multirow{2}{*}{ Sexo } & & \multicolumn{3}{|c|}{ Servicio } & \multirow{2}{*}{ Total } \\
\hline & & Medicina & Ginecologia & Cirugía & \\
\hline \multirow[t]{2}{*}{ Femenino } & $\mathrm{N}^{\circ}$ & 9 & 23 & 9 & 41 \\
\hline & $\%$ & $13,8 \%$ & $35,4 \%$ & $13,8 \%$ & $63,1 \%$ \\
\hline \multirow{2}{*}{ Masculino } & $\mathrm{N}^{\mathrm{o}}$ & 14 & 0 & 10 & 24 \\
\hline & $\%$ & $21,5 \%$ & $0 \%$ & $15,4 \%$ & $36,9 \%$ \\
\hline \multirow{2}{*}{ Total } & $\mathrm{N}^{\circ}$ & 23 & 23 & 19 & 65 \\
\hline & $\%$ & $35,4 \%$ & $35,4 \%$ & $29,2 \%$ & $100,0 \%$ \\
\hline
\end{tabular}




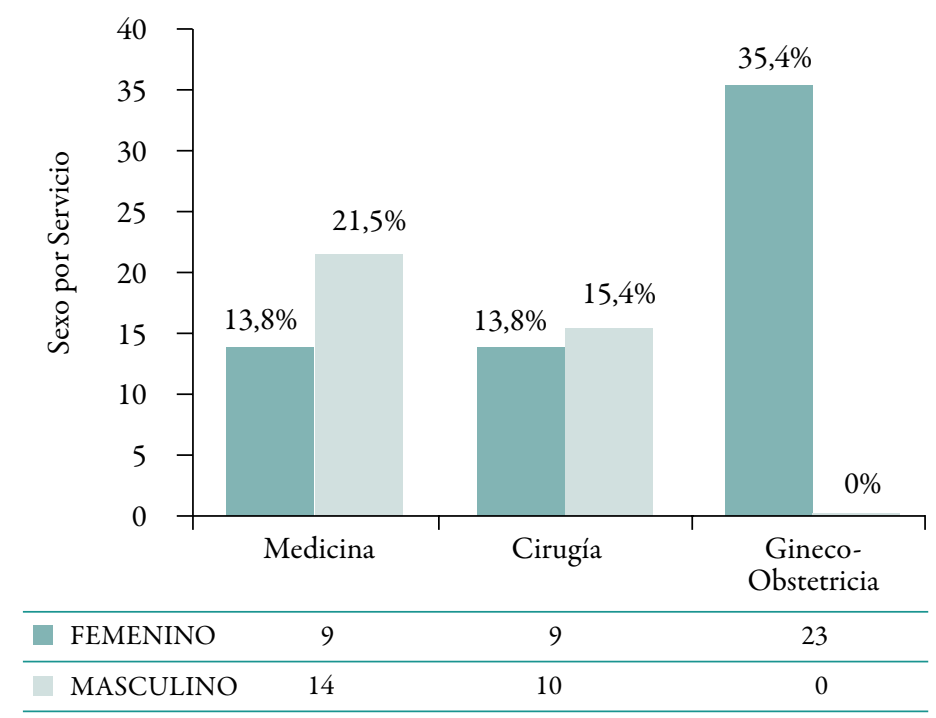

Figura 1. Pacientes por servicio.

Frecuencia de la percepción del cuidado humanizado

Tabla 2. Percepción del cuidado de enfermería.

\begin{tabular}{|c|c|c|c|c|c|c|c|c|c|}
\hline \multirow{2}{*}{ Indicadores } & \multicolumn{2}{|c|}{ Nunca } & \multicolumn{2}{|c|}{ Algunas veces } & \multicolumn{2}{|c|}{ Casi siempre } & \multicolumn{2}{|c|}{ Siempre } & \multirow{2}{*}{$\begin{array}{c}\text { Total } \\
\%\end{array}$} \\
\hline & $\mathbf{F}$ & $\%$ & $\mathbf{F}$ & $\%$ & $\mathbf{F}$ & $\%$ & F & $\%$ & \\
\hline 1. Sentimiento del paciente & 11 & 16,9 & 29 & 44 & 11 & 16 & 14 & 21 & 100 \\
\hline 2. Dar apoyo emocional & 5 & 7,7 & 31 & 47,7 & 20 & 30,8 & 9 & 13,8 & 100 \\
\hline 3. Dar apoyo físico & 11 & 16,9 & 23 & 35,4 & 12 & 18,5 & 19 & 29,2 & 100 \\
\hline 4. Cualidades del hacer de la enfermera & 14 & 21,5 & 21 & 32,3 & 15 & 23,1 & 15 & 23,1 & 100 \\
\hline 5. Proactividad & 19 & 29,2 & 20 & 30,8 & 11 & 16,9 & 15 & 23,1 & 100 \\
\hline 6. Empatía & 12 & 15,8 & 34 & 52,3 & 9 & 13,8 & 10 & 15,4 & 100 \\
\hline 7. Priorizar al ser cuidado & 24 & 36,9 & 20 & 30,8 & 11 & 16,9 & 10 & 15,4 & 100 \\
\hline 8. Disponibilidad para la atención. & 15 & 23,1 & 36 & 55,4 & 9 & 13,8 & 5 & 7,7 & 100 \\
\hline TOTAL & 145 & 24,8 & 232 & 39,7 & 106 & 18,1 & 102 & 17,4 & 100 \\
\hline
\end{tabular}

Frecuencia de la percepción del cuidado por servicio

En la figura 2 se evidencia que de un total de 65 pacientes encuestados, en los servicios de medicina (1) y cirugía (2) el 15\% manifiesta que algunas veces las enfermeras atienden sus necesidades básicas, mientras que en el servicio de gineco-obstetricia (3) el 8,5\% manifiesta que siempre es atendido.
En la figura 3 se muestra que de un total de 65 pacientes encuestados, en el servicio de cirugía (2) el $8,45 \%$ manifiesta que siempre percibe que las enfermeras demuestran conocimientos y habilidades profesionales, mientras que en los servicios de medicina (1) y gineco-obstetricia (3) el 5,2\% manifiesta que algunas veces percibe esta característica. 


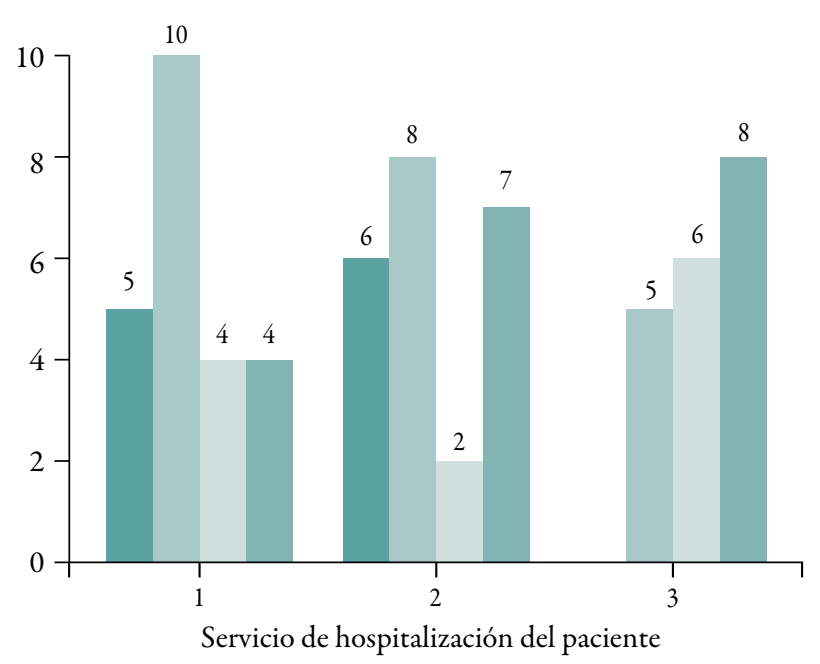

Figura 2. Servicio atiende sus necesidades básicas.

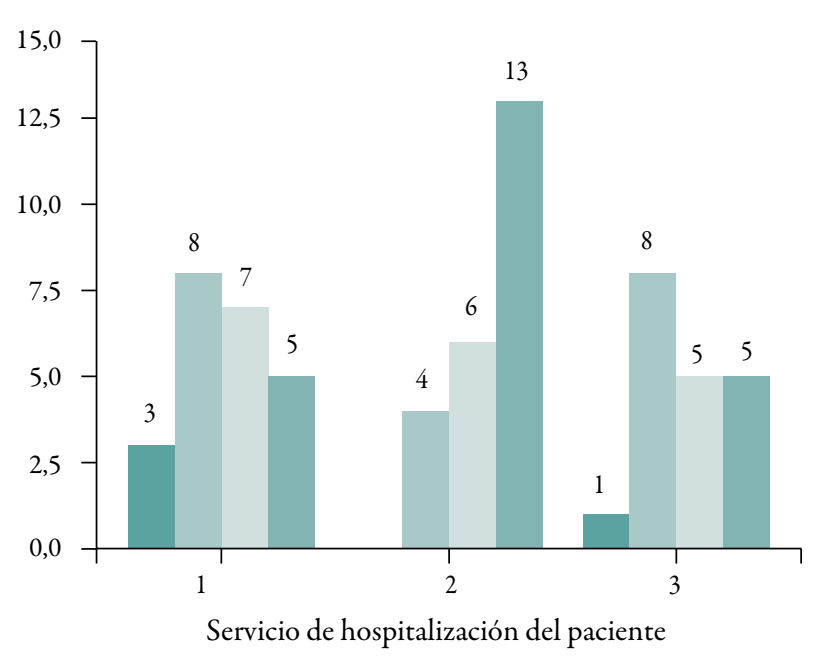

Figura 3. Servicio demuestra conocimientos y habilidades profesionales.

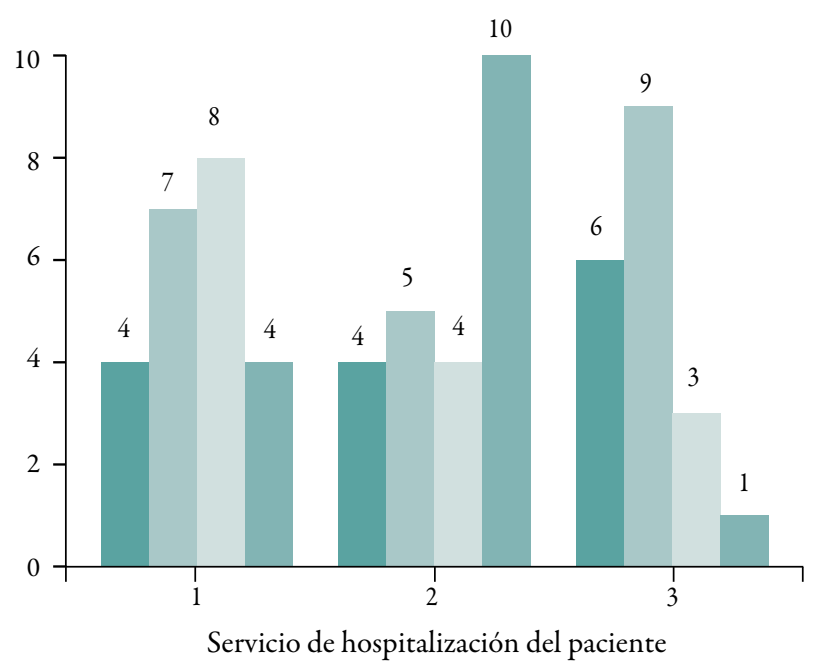

Figura 4. Servicio enfermero le explica el procedimiento.

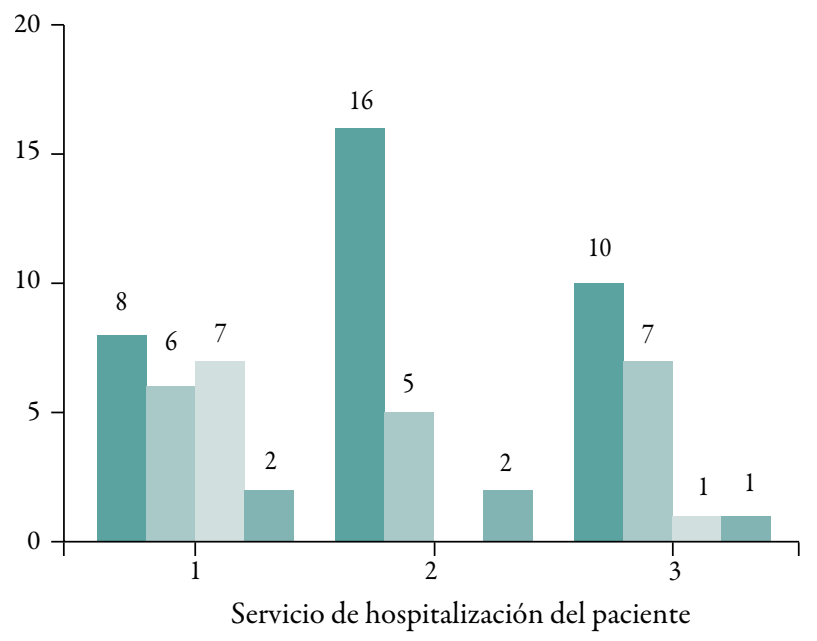

Figura 5. Servicio enfermero se pone en su lugar para comprenderlo.

En la figura 4 se denota que de un total de 65 pacientes encuestados, en el servicio de cirugía (2) el 8,45\% manifiesta que siempre el profesional de enfermería les explica el procedimiento antes de realizarlo, en el servicio de gineco-obstetricia (2) el 5,9\% manifiesta que algunas veces lo hace, y en el servicio de medicina (1) el 5,2\% manifiesta que casi siempre realiza esta acción.

En la figura 5, se evidencia que de un total de 65 pacientes encuestados, el 10,4\% en el servicio de cirugía (2), el 6,5\% en gineco-obstetricia (3) y el 5,2\% en medicina (1) manifiestan que nunca perciben empatía y comprensión.

En la figura 6 se evidencia que de un total de 65 pacientes encuestados, en el servicio de gineco-obstetricia (3) el 5,9\% manifiesta que algunas veces el profesional de enfermería respeta su intimidad, en el servicio de Cirugía (2) el 5,9\% refiere que siempre y casi siempre lo hace, y en el servicio de medicina (1) el 5,2\% manifiesta que nunca la respeta.

En la figura 7 se muestra que de un total de 65 pacientes encuestados, el 9,1\% en el servicio de cirugía (2), el 7,8 \% en medicina (1) y el 6,5\% en gineco-obstetricia (3) manifiestan que algunas veces el profesional de enfermería responde con rapidez a su llamado. 


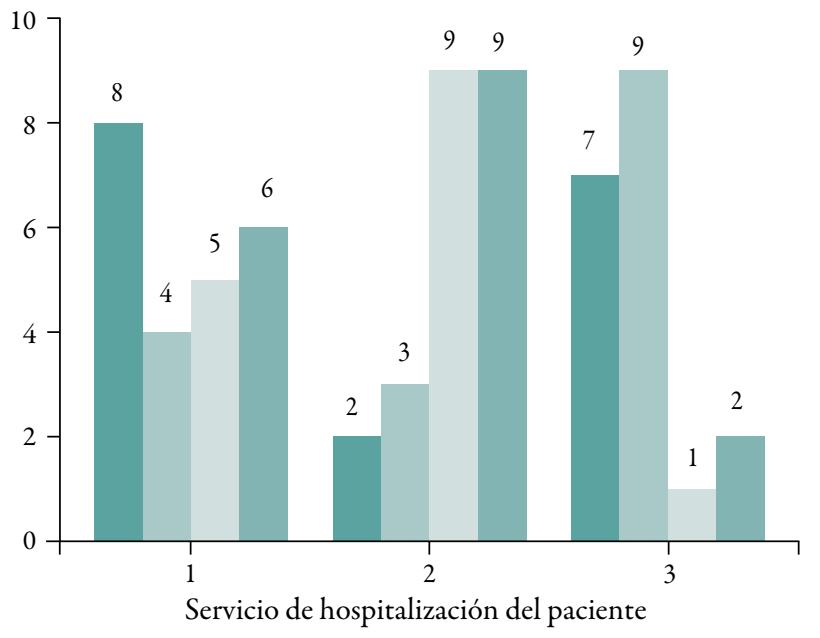

Figura 6. Servicio enfermero respeta su intimidad.

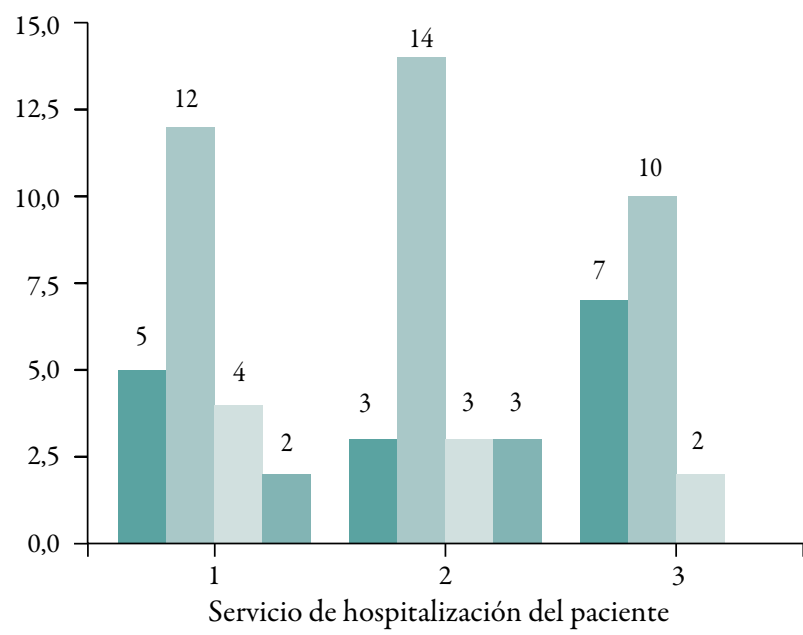

Figura 7. Servicio enfermero responde con rapidez al llamado.

En la figura 8 se muestra que de un total de 65 pacientes hospitalizados, el 7,8\% en el servicio de gineco-obstetricia (3), el $6,5 \%$ en cirugía (2) y el 5,9\% en medicina (1) manifiestan que algunas veces el profesional de enfermería se preocupa por su estado de ánimo. En la figura 9 se evidencia que de un total de 65 pacientes hospitalizados, en el servicio de cirugía (2), un 9,1\% manifiesta que siempre el profesional de enfermería muestra respeto hacia el paciente, en el servicio de medicina (1) un 6,5\% afirma lo mismo, y en gineco-obstetricia (3) un 5,9\% refiere que algunas veces el profesional de enfermería muestra esta actitud.

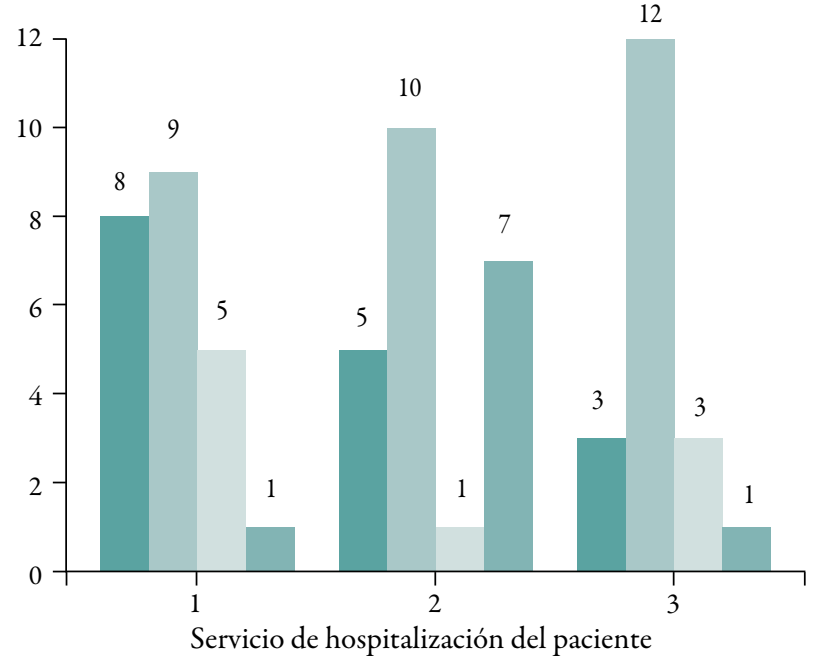

Figura 8. Servicio enfermero se preocupa por su estado de ánimo.

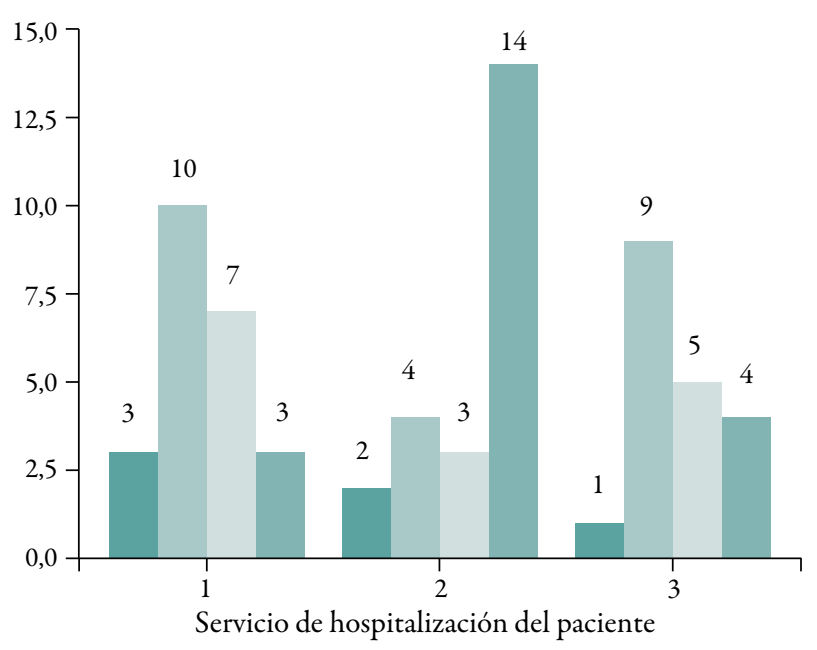

Figura 9. Servicio enfermero muestra respeto hacia el paciente.

\section{DISCUSIÓN DE RESULTADOS}

Los resultados encontrados apoyan el hecho de que las características del cuidado humanizado tiene una percepción significativa en los pacientes hospitalizados de los servicios de medicina, cirugía y gineco-obstetricia del Hospital Gustavo Lanatta Luján de EsSaludHuacho.

Lo evaluado en el presente trabajo concuerda con Gonzales J, Quintero D. (2009), en el sentido de que concluye que un $36,6 \%$ de los pacientes califican como negativa la relación 
que la enfermera establece con el paciente, puesto que al brindar la atención no se identifica adecuadamente, por lo que se recomienda crear conciencia de la necesidad de presentarse antes de realizar un procedimiento, pues esto ayuda a establecer una relación de confianza. El 40\% refiere que no existe empatía entre enfermera y paciente, por lo que se recomienda a la enfermera mostrar verdadero interés por el estado emocional y físico del paciente a fin de que este sienta que el personal de enfermería comprende lo que le está pasando (4). Resulta evidente que para la enfermería, la práctica de un cuidado humanizado es imprescindible durante el proceso de hospitalización necesario para la atención de una enfermedad. Por su parte, las variables de género y grupo etáreo estudiadas no reportaron significancia estadística en cuanto a los niveles de percepción del cuidado humanizado. De otro lado, se reporta que las categorías de Watson relativas al cuidado humano solo algunas veces son observadas por los usuarios de salud en el Hospital EsSalud de Huacho. Esto último difiere con los resultados de Romero V., Cabeza B., Sainz J., San Marcos M., Carretón L., Escalante S., et al. (2006), quienes informan que un $94 \%$ de las enfermeras brinda una atención rápida y apoyo emocional, inspira confianza, despeja dudas del paciente y brinda una información adecuada, y que el $97 \%$ de pacientes ha sido acompañado por las enfermeras en momentos de duda y por lo tanto siente confianza en este personal (99\%), con lo que se concluye que la calidad de la atención brindada es fruto del esfuerzo, la perseverancia, la constancia y la capacidad de las personas que gestionan el sistema sanitario, y que lo hacen sabiendo que el primer activo de una organización son las personas (1).

Ante ello, es necesario realizar un proceso comparativo entre las instituciones de salud de la región donde se ubica la ciudad de Huacho, a fin de verificar el trato brindado no solo en las salas de hospitalización sino en los diferentes ámbitos de asistencia al ser humano.

\section{CONCLUSIONES}

\section{Conclusiones parciales}

1. Los profesionales de enfermería de los servicios de hospitalización de cirugía, medicina y gineco-obstetricia, de manera global, no están brindando un cuidado humanizado a los pacientes, pues el estudio muestra que el 24,7 \% de ellos nunca percibe este cuidado, en tanto que el $39,7 \%$ solo lo percibe algunas veces. Esto prueba la hipótesis general planteada en la investigación. Asimismo, se concluye que el profesional de enfermería no brinda una buena atención, lo que contribuye a incrementar notoriamente la insatisfacción del paciente.

2. Los indicadores obtenidos en relación con el cuidado percibido por el paciente muestran los siguientes resultados: el 55,4\% de pacientes declara que la categoría disponibilidad para la atención es percibida solo algunas veces, el 52,3\% afirma que la categoría empatía es percibida algunas veces, el $44,7 \%$ considera que el apoyo emocional se ofrece algunas veces, el 44,6\% dice que los sentimientos del paciente son considerados algunas veces, el $36,9 \%$ afirma que la priorización del cuidado nunca es considerada, y el $35,4 \%$ informa que el apoyo físico es ofrecido algunas veces. $\mathrm{Si}$ a esto agregamos que las cualidades del quehacer de enfermería y la proactividad son en general percibidas solo algunas veces por el paciente, podemos deducir en consecuencia que el personal de enfermería debe poner más interés y entrega en su trabajo, así como en la relación que establece con el paciente, puesto que tales aspectos constituyen factor importante para alcanzar la pronta recuperación del mismo así como su incorporación a la vida laboral 
Conclusión general

Se determinó que existe una percepción muy baja del cuidado que realiza el profesional de enfermería en favor de los pacientes, percepción basada en el hecho de que solo algunas veces se aplican los criterios para un cuidado humanizado y una adecuada calidad en la atención.

\section{RECOMENDACIONES}

1. Concienciar al personal profesional de enfermería sobre la importancia del cuidado humanizado e individual del paciente y del trato que merece como ser humano único e irrepetible.

2. El profesional de enfermería debe mantener una comunicación fluida para obtener información oportuna y eficaz sobre el paciente a fin de lograr la recuperación óptima del mismo.

3. Se recomienda practicar y cumplir el principio bioético de autonomía, respetando las decisiones del paciente en todo momento del cuidado.

4. Los docentes de la Facultad de Ciencias de la Salud deben enfatizar en la formación integral del futuro profesional, especialmente en la práctica de los principios bioéticas, a fin de brindar una formación basada en valores y rescatando el aspecto humanístico, espiritual y transpersonal en la formación de los futuros profesionales de la salud.

5. La Universidad debe promover investigaciones sobre calidad de atención para proponer mejoras en la formación académica de los profesionales de la salud.

\section{REFERENCIAS BIBLIOGRÁFICAS}

1. Romero V., Cabeza B., Sainz J., San Marcos M., Carretón L., Escalante S., et al. (2006) Calidad asistencial en el sistema sanitario. Madrid.

2. Muñoz Y., Coral R., Moreno D., Pinilla D., Suárez Y. (2009) Significado del cuidado humanizado en egresadas de la facultad de enfermería. Repert. med.; 18(4):246-250.

3. Poblete M., Valenzuela S. (2007) Cuidado humanizado: un desafio para las enfermeras en los servicios hospitalarios. Acta Paul Enferm; 20(4):499-503.

4. Gonzales J., Quintero D. Percepción del cuidado humanizado en pacientesqueingresan al servicio de hospitalización de urgencias de gineco-obstetricia de una institución de II y III nivel de atención (tesis para optar el título de licenciado en enfermería). Bogotá, Pontificia Universidad Javeriana, 2009.

5. Ley general de Trabajo del Enfermero Peruano No 27669 y su Reglamento (decreto supremo 004-2002-sa). Lima, junio, 2002.

6. Rivera L., Triana A. (2007) Cuidado bumanizado de enfermeria: visibilizando la teoría y la investigación en la práctica, en la clinica del country. Enferm.; 10(4):15-21.

7. González G., Bracho de L., Zambrano de G,, Marquina M,, Carmen A. (2004) Cuidado bumano como valor en el ejercicio de los profesionales de la salud.

8. Bautista L,, (2008) Percepción de la calidad del cuidado de enfermería en la empresa social del Estado Francisco de Paula Santander. Redalyc; 8 (1): 74-84.

9. Escobar D. (2007) El poder del cuidado humano amoroso en la enfermería. Revista Latinoamericana Enferm. gen; 15(4): 1-08.

10. Torres J. (2008) Estrés laboral y calidad del cuidado de la enfermera(o) en hospitales del MINSA. (Tesis para optar el grado de doctor 
en educación en la Universidad de Murcia). Murcia: Universidad de Murcia.

11. Ariza C, Daza R. (2008) Calidad del cuidado de enfermería al paciente hospitalizado en un hospital de tercer nivel de Bogotá. Rev. Enferm. 8 (3): 8-14.

12. Alvis T, Moreno M, Muñoz S. (2002) "Experiencia de recibir un cuidado de enfermería humanizado en un servicio de hospitalización". En: el arte y la ciencia del cuidado. Bogotá: unibiblos. P. 207-216.

13. Colegio de Enfermeros del Perú. (2002) Código de ética y deontología. Lima.
14. Wong Y, García L, Rodríguez E. (2008) Percepción de la calidad de la atención de los servicios de salud: perspectiva de los usuarios (tesis para optar el grado de máster en salud pública en la Universidad Nacional Autónoma de Nicaragua). Matagalpa, Universidad Autónoma.

15. Hernández L., Zequeira D., Miranda A. (2010) La percepción del cuidado en profesionales de enfermería. Revista cubana de enfermería; 26 (1):30-41.

16. Torres A. (2001) Experiencias de conceptualización de enfermería. La experiencia de conceptualización en la Facultad. Editorial Ceja, Bogotá. 